\title{
Determinants of food safety practices among food handlers in selected food establishments
}

\author{
Abdu Oumer \\ ${ }^{1}$ College of Health sciences and Medicine, Department of Public Health, Wolkite University, Ethiopia
}

\begin{tabular}{l} 
Article Info \\
\hline Article history: \\
Received Feb 20, 2019 \\
Revised Mar 27, 2019 \\
Accepted Apr 10, 2019 \\
\hline
\end{tabular}

\section{Keywords:}

Ethiopia

Food Eestablishments

Food Handlers

Food Safety

\begin{abstract}
Despite great efforts against foodborne diseases, the occurrence of these problems remains a public health issue in developed and developing countries. This study was to assess determinants of food safety practice among food handlers in selected food establishments in Dire Dawa City administration, in 2017. Cross sectional survey was conducted among 356 food handlers from the selected food establishments in different categories. The CODEX food safety questionnaire was used to assess food safety knowledge (23 questions), attitude (six questions) and practice using interview and observation checklists (15 points). Satisfactory practice was defined as those who practice $70 \%$ of food safety measures correctly. Frequency, percentage, mean, binary logistic regression with a crude odds ratio and adjusted odds ratio (AOR) at 95\% confidence interval were calculated. Out of the total study subjects, 354 food handlers participated in this study with $99.4 \%$ response rate. A total of 191 food handlers, $54.0 \%$ were female with overall mean age of 29.5 years. About 129 (36.4\%), 194 (54.8\%) and 106 (29.9\%) had adequate knowledge, positive attitude and satisfactory food safety practice. Having food safety training $(\mathrm{AOR}=2.0)$, with adequate knowledge $(\mathrm{AOR}=2.83)$, positive attitude ( $A O R=2.09)$, presence of food safety guideline $(A O R=2.23)$ and supervision $(A O R=2.07)$ were significant predictors of satisfactory food safety practices. In general food safety/hygienic practices of food handlers was found to be low. Having adequate knowledge, positive attitude, presence of food safety guideline and formal food safety training were significant predictors of food safety practices among food handlers.
\end{abstract}

Copyright (c) 2019 Institute of Advanced Engineering and Science. All rights reserved.

\section{Corresponding Author:}

Abdu Oumer,

Department of Public Health,

Wolkite University, Ethiopia.

Email: phnabu@gmail.com

\section{INTRODUCTION}

Food safety can be defined when all measures taken so that food is consumed in the usual manner do not cause harms to human health and wellbeing. Poor food safety practice is one of the major contributors of food illness worldwide [1-2]. There are four critical food safety factors that are critical to maintain food safe and prevent foodborne diseases (FBDs). These are contamination/cross contamination, personal health and hygiene and temperature control. Food handlers and food workers are one of the critical points for the introduction of foodborne pathogens or hazards to the consumers [3]. The impact of this will be significant in setting where there are a number of customers in cafes, restaurants, hotels and others to be served per day, in that the target to be affected will be higher [1].

Thus unsafe food handling practices by food handlers and infected (carrier) food handlers are the primary source of infection and introduction of hazards to food. Food handlers' knowledge towards food safety is critical to handle food safely and to restrict themselves in preparation of food when they are symptomatically ill or carrier sate with the ultimate goal of protecting its customer [4]. It continues to be a public health problem 
globally, because foodborne illnesses are widespread affecting every segment of the population. Unfortunately, only few percent of cases of foodborne diseases are reported. But foodborne diseases are major causes of morbidity, Death with greater impact on social and economic development. As of 2015 World Health Organization (WHO) estimates, FBD had great contribution to the global burden of disease. Additionally Food safety is of a great concern to tourists and meet the international regulatory requirements to world trade greatly reducing the economic development of region [1].

FBDs are estimated to account for the loss of about 33 million disability adjusted life years (DALYs) globally. Among the different segments of community, FBD affects about 40\% children as of 2010 [5]. Out of all continents, Africa and Asia is the carrier of the biggest burden of FBD worldwide [1]. An estimated 91 million people in Africa Region are affected each year from foodborne diseases; which results in 137,000 deaths, accounting for a third of the global death burden. In Africa, about $70 \%$ of the burden of foodborne diseases is due to diarrheal diseases, followed by other acute and chronic complications of FBDs [6]. Poor food safety practice by food handlers have been associated with lack of formal training on food safety/hygiene, educational level, settings of the food establishments, knowledge and attitude on food safety measures and others [6]. But having knowledgeable food handlers may not necessarily associated with good food safety practice, in that food handlers are prone to optimistic bias. Thus those who have higher risk perception are not necessarily knowledgeable or put their knowledge into practice in that know do gap might be problem [7].

A study done in Northern Ethiopia showed that, 53\% and $72 \%$ of food handlers working in street vendors and other food establishments had good food safety practice respectively. Having food safety training (AOR=6.7, 6.7, 95\% CI=1.8-25), wearing a gown during food handling ( $\mathrm{AOR}=19,95 \% \mathrm{CI}=6.8-51)$ and with regular health follow ups (AOR=5.2, 95\% CI=2.1-13) were significant predictors of satisfactory food safety practices [8]. Also in Ethiopia, about 52.5\% of food handlers had proper food handling practices with those married ( $\mathrm{AOR}=7.52$ ), monthly income ( $\mathrm{AOR}=0.395)$, knowledge about food handling ( $\mathrm{AOR}=1.69)$, presence of body washing facility in the establishment $(\mathrm{AOR}=1.89)$ and presence of separate room for dressing $(\mathrm{AOR}=1.97)$ were significant predictors of good food handling Practices [9].

In addition, shortage of regular food safety training packages and certification, problems with organization of equipment, lack of knowledge and supervision were related with poor food handling practices [10]. So food workers have a substantive role in ensuring food safety protocols and ultimately protecting the customers. Food handlers with poor food handling and preparation practices including maintenance of safety protocols in personal hygiene at the appropriate times and using appropriate methods, can contaminate food and transmit FBD to large number of potential customers [10]. Studies conducted in Ethiopia also depicted that, food safety practices of food handlers were low and caused high burden on morbidity and mortality from FBDs [8-9, 11-12].

\section{RESEARCH METHOD}

\subsection{Study setting}

The current survey was conducted in Dire Dawa city, one of the city under the federal government from May 24 - June 20, 2017. It is found in the eastern Ethiopia around $515 \mathrm{~km}$ from the capital city of Ethiopia, Addis Ababa. In the administration, there are 38 rural and nine urban lowest administrative units called Kebeles. As of 2015, it has a total population of 453,000 of whom 227,406 (50.2\%) male and 225,594 (49.8\%) female. As of city culture and tourism Bureau report, there were 69 hotels, 98 restaurants, 112 cafeterias and 122 butcher shops that were registered and regulated by the respective office. The estimated average number of food handlers in each hotel, restaurant, cafeteria and butcher shops were approximately 12, 10, 7 and 5 per facility respectively in 2015 [8].

\subsection{Study design and population}

Facility based survey was conducted among randomly selected food handlers working in selected food establishments in Dire Dawa city administration, in 2017. Food handlers who had responsibility to handle packaged/unpackaged food, food equipment and utensils or food contact surfaces employed in selected hotels, restaurants, cafeterias and butcher shops were interviewed and observed for food safety practices.

\subsection{Sample size determination}

Proportion estimate with sample size calculation for single population proportion was used to estimate the minimum sample size for this study. Thus using $30.3 \%$ prevalence of proper food safety practice among food handlers from similar study [9] with 5\% margin of error (d) and 10\% non-response rate. The minimum study size for this studywas calculated as follows using the above statistics. 


$$
n=(Z \boldsymbol{\alpha} / \mathbf{2})^{2} \boldsymbol{p}(\mathbf{1}-\boldsymbol{p})
$$

$d^{2}$

Where, $\mathrm{n}=$ sample size

$\left(Z_{\alpha / 2}\right)=$ confidence level; taken $95 \%$ level of confidence interval,

$\mathrm{P}=30.3 \%, \mathrm{q}=1-\mathrm{p}=69.7 \%[9]$

$\mathrm{d}=$ margin of error $(0.05)$

Considering an estimated non-response (incompleteness of the data) as $10 \%$, the minimum sample size calculated was 356. And using sample size for sex of food handlers as determinants of food safety practice, the sample size was calculated as 138 using estimates of parameters from study [11]. Thus the larger final sample size of 356 was used.

\subsection{Sampling procedures}

They were total 3,202 food handlers ( 828 from hotels, 980 from restaurant, 784 from cafeterias and 610 from butcher shop) employed and working in 401 reported total food establishments (i.e. 69 Hotels, 98 restaurants, 112 cafeterias and 122 butcher shops) in the city. Stratified sampling with proportional allocation of the total sample size to selected Hotels, cafeterias, Butchers and Restaurants was conducted. Then a simple random sampling technique was used to select the samples from each food establishments according to their sample size. All the selected food establishments were included in the observation check list for food safety practice assessment.

About 31, 24, 12 and 37 cafeterias, restaurants, hotels and butcher shops were randomly selected out of the total 69 hotels, 98 restaurants, 112 cafeterias and 122 butcher shops. So as to increase the representativeness of the samples from sub samples, proportion to the number of food handlers from each selected was done. Then after from the selected food establishments, a total of 98, 109, 65 and 84 food handlers were selected from the above hotel, restaurants, cafeterias and butcher shops.

\subsection{Variables}

Food safety practice of food handlers was the dependent variable while socio demographic characteristics (sex, age, marital status, educational status, monthly income, service year, training on food safety/hygiene, knowledge, attitude of food handlers to food safety practices, availability of guidelines for food safety/hygiene were predictor variables of this study.

\subsection{Data collection instrument and techniques}

Five graduates of grade 10th and 12th was recruited and trained. Data were collected by face to face interview using a standardized questionnaire and contextualized adopted from CODEX [9]. The questionnaire was translated to local language convenient to participants. In addition, the existing actual food safety practice of food handlers was observed using an observation check list. The observation was made by randomly selecting one food handler (cooker) (using lottery method) from each selected food establishment. Twenty four (24) knowledge, six attitude and fifteen food safety related practice questions were included in the interview.

In knowledge assessment, correct responses to food safety practice were given a score of one and incorrect responses were scored as zero. While for attitude assessment a sets of likert scale was used. Then the knowledge and the attitude scores were added up, then scores above seventy percentile was taken as a cutoff point to classify each score in to adequate and inadequate measures. Adequate knowledge was assessed if the respondent's knowledge score summed up is above seventy percentile (score $>=15$ ) for critical food safety factor related questions by using the CODEX food hygiene and safety principles [13]. Positive attitude was assessed if respondents score for attitude related questions is above seventy percentile for summed up knowledge score (score $>=18$ ). Respondents' score for food hygiene practice related questions of greater than or equal to seventy percentile (score>=36.5) was considered as cuts of point for satisfactory food safety practice [9].

The overall Cronbach's alpha level is 0.7 . It is fair according to the study. While in order to assess the inter rater reliability Cohen's kappa was calculated for each item from two independent data collectors. For those of the items for attitude the Cohen's kappa value was about 0.68 showing substantial agreement between observers. In order to assess food handlers' attitude towards food safety practices and principles, Likert scale questions were used. To assess the internal consistency of the questions to measure attitude reliability analysis was done with Cronbach's alpha level of 0.7 which shows fair level of consistency. Additionally inter rater reliability with Cohen's kappa was calculated for each item from two independent data collectors. For those of the items for attitude the Cohen's kappa value was about 0.68 showing substantial

Determinants of food safety practices among food handlers in selected food establishments (Abdu Oumer) 
agreement between observers. Data collectors were selected and trained for three days on the overall data collection processes, procedures, and techniques for probation in order to get accurate data. Pretest was conducted before data collection in some food establishments out of the study populations and amended accordingly.

To assess the inter rater consistency/reliability of data collectors on food safety practices of food handlers, preliminary test was conducted before the actual field work during pretest period. Thus data collectors were instructed independently to assess the food safety practices of twelve selected food handlers using separate check list. Then the inter rater reliability was constructed and conducted. Those with disagreeing assessment as compared to the standard/Reference subject/were given a second chance to take training and improve their performance at least 0.6 (substantial agreement). Then they are involved in the actual data collection, unless they will not be involved in the study.

\subsection{Biases}

Since this study involved direct observation of food handlers' food safety practice, observers' bias and Howthrone effect due to the presence of the observer are the two common problems in this study. It is evident from study [14], that those food handlers with auditors had higher dispense rate of hand washing materials than counterparts. In order to minimize the biases the first thing, data collectors were trained on the objective criteria to assess the food safety practices of food handlers through observation of the reality with the guide of the checklists and its options. The data collectors again informed the food handlers that this observation is not a supervision but it is attempt to gain insight on food safety practice and it did not have any punishment or rewards. Also the information collected is strictly confidential in that no personal identifier is not collected. Before the actual data collection the instrument was being checked by panels of experts with a focus on face validity and content validity of the tools and necessary amendments was done.

\subsection{Data processing and analysis}

Data entry and analysis was performed using SPSS version 20. Bloom's cut off points was used to determine practice level. To assess level of practice, 15 questions were prepared by using the CODEX food hygiene and safety principles. A score of one to three (one for never, two sometimes and 3 for always) was given for each food safety practice parameters. Level of practice was classified into unsatisfactory practice (score less than 36.5) and satisfactory practice (score above or equals to 36.5 of their overall practices score).

Descriptive statistics like frequency, percentage, mean, median, standard deviation and others. Bivariate logistic regression analysis with crude odds ratio, $\mathrm{P}$-values of 0.05 was used to declare significant association food safety practice. In Multivariate analysis, adjusted odds ratio (AOR) with 95\% confidence interval (CI) was calculated for selected independent variables. To assess the reliability of the tool and the inter rater reliability, cronbanch's $\alpha$, Cohen's kappa was calculated. Additionally as measure of model fitness, $\chi 2$ statistics with coefficient of determination (R2) and Hosmer and Lemeshow's test was calculated.

\subsection{Ethical consideration}

Ethical clearance was obtained from health Science College, Institutional Health Research ethical review committee. Permission was obtained from Dire Dawa health office and cultural tourism Bureau. Written Informed consent was obtained from owners/managers of the establishments but verbal from study subjects. Each respondent were assured that the information provided was kept confidential and used only for the purpose of this research.

\section{RESULTS AND DISCUSSIONS}

\subsection{Results}

\subsubsection{Socio-demographic characteristics of food handlers}

Out of the total 356 questionnaires distributed the response rate of 99.4\% (354). The age of food handlers was ranging from 17 to 60 with mean age was 29.5 (+SD 7.1) years. About $191(54.0 \%)$ were female while half, $177(50.0 \%)$ of the respondents were single. Regarding to education status of the respondent, more than one third 139 (39.3\%) of them attended primary education (grade 1-8). Socio-demographic characteristic of food handlers among food handlers is presented in Table 1. 
Table 1. Socio-demographic characteristic of food handlers among food handlers

\begin{tabular}{cccc}
\hline Variables & & Frequency & Percentage (\%) \\
\hline Sex $(\mathrm{n}=354)$ & Female & 191 & 54.0 \\
& Male & 163 & 46.0 \\
& $15-20$ & 21 & 5.9 \\
& $21-24$ & 70 & 19.8 \\
Age $(\mathrm{n}=354)$ & $25-29$ & 110 & 31.1 \\
& $30-34$ & 70 & 19.8 \\
& $35-39$ & 47 & 13.3 \\
& $40-44$ & 21 & 5.9 \\
& $>45$ & 15 & 4.2 \\
Marital status & Single & 177 & 50.0 \\
$(\mathrm{n}=354)$ & Married & 146 & 41.3 \\
& Divorced & 22 & 6.2 \\
& Widowed & 9 & 2.5 \\
Educational & Illiterate & 8 & 3.3 \\
status & Primary education & 139 & 39.3 \\
$(\mathrm{n}=331)$ & Secondary education & 121 & 34.2 \\
& Diploma & 47 & 13.3 \\
Monthly & Degree or above & 16 & 4.5 \\
Income, ETB & $<600$ & 50 & 14.1 \\
(n=354) & 600-1000 & 149 & 42.1 \\
& $>1000$ & 155 & 43.8 \\
\hline
\end{tabular}

\subsubsection{Working situation of respondents}

There are totally 3,202 food handlers employed in the selected 354 food establishments of Dire Dawa city administration. Almost one thirds of the food handlers had work experience of 3 to 4 years. Majority 142 $(40.1 \%)$ work as waiter/waitress followed by cooker 126 (35.6\%). Work experiences of the respondents were ranging from one to two years, 128 (36.2) More than one third of respondents, 139 (39.2\%) had food safety training at any point in time as shown in Table 2.

Table 2. Work experience and occupational roles of food handlers

\begin{tabular}{cccc}
\hline & & Frequency & Percentage \\
\hline \multirow{2}{*}{ Work experiences } & 1-2 years & 128 & 36.2 \\
$(\mathrm{n}=354)$ & 3-4 years & 116 & 32.8 \\
& $>5$ & 110 & 31.1 \\
& Waiter/ess & 142 & 40.1 \\
Role & Cooker & 126 & 35.6 \\
& Manager & 42 & 11.9 \\
& Others & 44 & 12.4 \\
\hline
\end{tabular}

\subsubsection{Knowledge and attitudes of food handlers towards food safety practice}

Bloom's cut off points was used to determine knowledge level using a total of 23 knowledge questions prepared from CODEX food hygiene and safety principles containing four food safety dimensions. Based on the sum scores level of knowledge was classified into two adequate knowledge (score above or equals to 15) and inadequate knowledge (score below 15). From 354 respondents, 200 (56.5\%) had adequate knowledge on transmission of foodborne diseases. More than half of the respondents, 272 (76.8\%) had good knowledge on personal health and hygiene. While $271(76.6 \%)$ and $113(32.0 \%)$ of food handlers had adequate knowledge on food contamination through time and temperature control respectively. Overall, $129(36.4 \%)$ of the respondents were found to have adequate knowledge on Food safety practice (overall score equals or above 36.5), while 225 (63.6\%) had poor knowledge as shown in Table 3. Based on the composite $194(54.8 \%)$ have positive attitude towards food safety practices (score greater than or equals to 18, of the attitude question and the remaining 160 (45.2\%) have negative attitude as shown in Figure 1.

Table 3. Knowledge of food handlers based on four categories of questions assessing food safety practices among respondents

\begin{tabular}{lcccc}
\hline \multicolumn{1}{c}{ Categories } & Adequate knowledge (\%) & \multicolumn{2}{c}{ Inadequate knowledge (\%) } \\
& Freq. & $\%$ & Freq. & $\%$ \\
\hline Food Safety Knowledge & & & & \\
Transmission of foodborne diseases & 200 & 56.5 & 154 & 43.5 \\
Personal health and hygiene & 272 & 76.8 & 82 & 23.2 \\
Contamination/cross contamination & 271 & 76.6 & 83 & 23.4 \\
Temperature control & 113 & 32.0 & 241 & 68.0 \\
Over all knowledge Level & 129 & 36.4 & 225 & 63.6 \\
\hline
\end{tabular}

Determinants of food safety practices among food handlers in selected food establishments (Abdu Oumer) 


\section{Food safety Attitude levels of Food handlers}

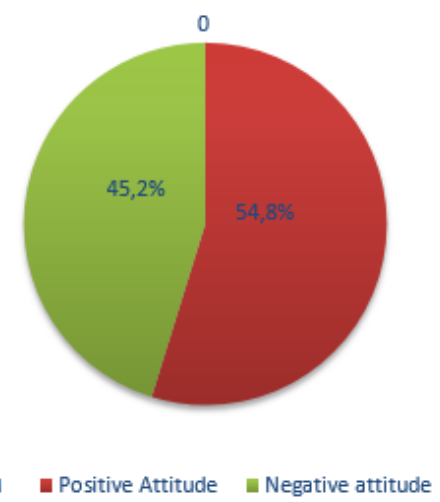

Figure 1. Food handlers' food safety attitude level among respondents

\subsubsection{Food safety practice of food handlers}

From 354 food handlers, 106 (29.9\%) food handlers had satisfactory food safety practices and the remaining $248(70.1 \%)$ had unsatisfactory food safety practices as shown in Table 4.

Table 4. Food practice level of food handlers towards food safety practice

\begin{tabular}{ccc}
\hline & Level of Food Safety Practice & Percentage \\
\hline Satisfactory Food safety practice & 106 & $29.9 \%$ \\
Unsatisfactory food safety Practice & 248 & $70.1 \%$ \\
\hline
\end{tabular}

Among the 354 food handlers, 279 (78.8\%) did not had long nails and majority 261 (73.7\%) wore clean uniforms. Moreover, $194(54.8 \%)$ wore head cap and did not wear hands jewellery while preparing foods were $258(72.9 \%)$. About 291 (82.2\%) did not show Sneezing/ coughing over uncovered foods of respondents. Near to three fourth 255 (72.0\%) wash hands after before handle food. Almost $278(78.5 \%)$ do not works with discharge from the nose and cut/any skin problem. From total of 354 interviewed food handlers, 243 (68.6\%) did not use same chopping board for raw and cooked food without cleaning as shown in Table 5.

Table 5. Observed food hygiene practice of food handlers in food establishments

\begin{tabular}{lccc}
\hline & & Freq. & Percentage \\
\hline Having long nails & Yes & 75 & 21.2 \\
& No & 279 & 78.8 \\
Wearing clean uniforms & Yes & 261 & 73.7 \\
& No & 93 & 26.3 \\
Wearing a head gear & Yes & 194 & 54.8 \\
Having jeweler while handling & No & 160 & 45.2 \\
foods & Yes & 96 & 27.1 \\
Sneezing/ coughing over uncovered & No & 258 & 72.9 \\
food & No & 63 & 17.8 \\
Washing hands after before & Yes & 251 & 82.2 \\
handing food & No & 99 & 72.0 \\
Working while having discharge & Yes & 76 & 28.0 \\
from the nose, & No & 278 & 78.5 \\
Working while having cut/any skin & Yes & 76 & 21.5 \\
problem & No & 278 & 78.5 \\
Using same chopping board for raw & Yes & 111 & 31.4 \\
and cooked food without cleaning & No & 243 & 68.6 \\
\hline
\end{tabular}

\subsubsection{Determinants of food safety practices among food handlers}

According to this data the food handlers status, socio-demographic characteristics on food safety practices, sex, education status, marital status and services years were not found be significantly associated with food safety practices in food handler. But the age of respondents, attend training on food safety practices, 
knowledge levels of food handlers, attitude level of food handlers, food safety/hygiene guidelines available for food handlers and supervision by owner/supervisor was found is significantly associated with food safety practices of food handlers. Advance in educational status of food handlers has shown better food safety practices than illiterates as shown in Table 6.

Table 6. Binary logistic regressions to identify factors associated with satisfactory food safety practice in selected food establishments

\begin{tabular}{|c|c|c|c|c|c|}
\hline \multirow[b]{2}{*}{ Variable } & \multicolumn{2}{|c|}{ Food safety practice } & \multirow[b]{2}{*}{ p-value } & \multirow[b]{2}{*}{$\begin{array}{c}\text { COR } \\
(95 \% \mathrm{CI})\end{array}$} & \multirow[b]{2}{*}{ AOR 95\% CI } \\
\hline & $\begin{array}{l}\text { Satisfactory } \\
\text { practices }\end{array}$ & $\begin{array}{l}\text { Unsatisfactor } \\
\text { y practices }\end{array}$ & & & \\
\hline \multicolumn{6}{|l|}{ Sex } \\
\hline Female & 55 & 136 & 0.61 & 1 & \\
\hline Male & 51 & 112 & & $1.13(0.71-1.78)$ & \\
\hline \multicolumn{6}{|l|}{ Education status } \\
\hline Illiterate & 7 & 24 & & 1 & \\
\hline Primary education & 39 & 100 & 0.536 & $1.34(0.53-3.35)$ & \\
\hline Secondary education & 38 & 83 & 0.340 & $1.57(0.62-3.96)$ & \\
\hline college diploma & 14 & 33 & 0.484 & $1.46(0.51-4.15)$ & \\
\hline Degree or more & 8 & 8 & 0.062 & $3.43(0.94-12.48)$ & \\
\hline \multicolumn{6}{|l|}{ Work experiences } \\
\hline 1-2years & 37 & 90 & & 1 & \\
\hline 3-4years & 30 & 86 & 0.57 & $0.85(0.48-1.49)$ & \\
\hline$>$ yyears & 39 & 71 & 0.30 & $1.34(0.77-2.31)$ & \\
\hline \multicolumn{6}{|l|}{ Training } \\
\hline yes & 49 & 66 & $0.000 *$ & $2.44(1.52-3.90)$ & $2.0(1.20-3.34)$ \\
\hline No & 57 & 182 & & 1 & 1 \\
\hline \multicolumn{6}{|l|}{ Knowledge level } \\
\hline Adequate knowledge & 61 & 68 & $0.000 *$ & $3.59(2.23-5.77)$ & $2.83(1.72-4.68)$ \\
\hline Inadequate knowledge & 45 & 180 & & 1 & 1 \\
\hline Attitude level & & & & & \\
\hline Positive attitude & 71 & 123 & $.003 *$ & $2.06(1.28-3.32)$ & $2.09(1.24-3.51)$ \\
\hline Negative attitude & 35 & 125 & & 1 & 1 \\
\hline \multicolumn{6}{|l|}{$\begin{array}{l}\text { Food safety/hygiene } \\
\text { guidelines availability }\end{array}$} \\
\hline Yes & 56 & 80 & $0.000 *$ & $2.35(1.48-3.75)$ & $2.23(1.33-3.72)$ \\
\hline No & 50 & 168 & & 1 & 1 \\
\hline
\end{tabular}

Four variables including having food safety training, knowledge, attitude and presence of food safety guideline were included in the multivariate binary logistic regression with Hosmer and Lemeshow goodness of fit of $0.61\left(\chi_{2}=6.44\right)$. Knowledgeable food handlers on food safety were found to practice good safety practices 2.8 times than heir counters $(A O R=2.8395 \% \mathrm{CI}=1.72-4.68)$. Similarly having positive attitude towards food safety practice had two fold more likely to have satisfactory food safety practices than food handlers with negative attitude about food safety practice (AOR=2.09 (1.24-3.51)). Food handlers working in food establishments that had food safety or hygiene guidelines were almost two times more likely to have satisfactory food safety practice than the others $(\mathrm{AOR}=2.23(1.33-3.72))$ as shown in Table 5 .

\subsection{Discussions}

In this study significantly low proportion of food handlers, $29.9 \%$ (95\% CI=25.1\%-34.7\%) food handlers had satisfactory food safety practices. While Malaysian study in 2009 showed almost more than average, 59.1\% had satisfactory practice [15]. Similarly study on 302 food handlers, half of food handlers had a poor food handling practice [11]. While survey conducted in Gondar Ethiopia in 2014, good food safety practice was reported among $30.3 \%$ of them [10]. This marked variations among different studies suggested that food safety practice among food handlers is not satisfactory and it is still low. It primarily emphasize the need for training, increase skills and motivations of food handlers so as to prevent the major burdens of foodborne illnesses [16]. As respondent do not tends to underestimate their practice in negative way, the likely chance of social desirability bias will be low.

This low level of food safety practice might be due to the fact that small proportion, $36.4 \%$ of food handlers had good knowledge and more importantly significant proportion of them also had negative attitude which might influence their actual practice $[1,9]$. In other similar finding showed average score of food safety practice (3.98 \pm 0.55 out of 10) [17]. While higher level of food safety practice was reported $(90 \%)$, attitude $(66 \%)$ and knowledge on food safety $(80 \%)$ [18]. So generally this shows there is a great variation in food safety practice in different setting. Finding from this study showed significant associations of knowledge 
levels, attitude level of food handlers, presence of food safety/hygiene guidelines available for food handlers were found be significantly associated with food safety practices.

Similarly having food safety training and better work experience practiced food safety measures more likely $[11,19]$. Additionally similar study also pinpoint that training on food safety is a good predictor of food handling practice ( $\mathrm{p}$ value $=0.035$ ) [17]. In addition food safety training significantly improves knowledge level of food handlers ultimately increasing their safer food handling practices [20-21]. This will give clue on the pertinent issue regarding food handlers with no work experience working with their formal educational, training makes them not to be updated on food safety measures. So scheduled formal food safety training with availability of food safety guidelines in kitchens are highly recommended for food establishments so to improve and implement the principles of Hazard Analysis critical contact points. This in turn helps Additionally food handlers with adequate knowledge about food safety practice were 2.823 times more likely to have satisfactory food safety practices (AOR=2.83 95\% CI:1.69-4.68). Ethiopian study from Dangila town, showed that good knowledge of food handlers towards food safety significantly predict proper food safety practices $(\mathrm{AOR}=1.69,95 \% \mathrm{CI}, 1.05-2.73) \quad[9]$ and in Mekele $(\mathrm{OR}=3.61,95 \% \mathrm{CI}=1.51-8.65$ (18) [22]. In both of the studies use almost similar tool in assessing the knowledge and practices of food handlers. Thus having technical knowledge on food safety and food preparation technique is very crucial to ensure the safety and health of customers [16, 23]. So this will urge food establishments to hire food handlers with god technical skills and graduates of accredited institutions than habitual workers.

More importantly having positive attitude regarding food safety practice significantly improved satisfactory food safety practices (AOR=2.09 95\% CI=1.24-3.51). Similarly another study in Gonder showed having positive attitude towards food safety practices increased proper food safety practices [9]. In general, good technical knowledge coupled with positive attitude and eagerness to apply the knowledge leads to good preparation and handling of food which ultimately decrease burden of foodborne illnesses in our community. This evident from behavioral change model that shows awareness, knowledge, attitude and putting the theory in to practice via behavioral changes. Thus improving the awareness level coupled with positive beliefs and attitudes towards a healthy behavior are crucial for promotion of proper food handling and ultimately can save morbidity and mortality.

The author's conclusion on this particular manuscript should be seen in balance with some limitations of the study. As study shown, the probability of Howthrone effects and some risks of the observer's bias will not be neglected at all [14, 24]. Even though this might have some risks of the social desirability bias and the aforementioned drawbacks, it came up with necessary and updated information regarding poor food safety practices of food handlers.

\section{CONCLUSION}

In general food safety/hygienic practices of food handlers is unsatisfactory. Having adequate knowledge, positive attitude, Presence of food safety guideline and formal food safety training were significant predictors of food safety practices of food handlers.

The city Culture, Hotel and Tourism shall have strengthened regular supportive supervision on food establishments including food safety training, legal enforcement on the presence of food safety guidelines and inspection. Food establishments should hire a qualified food handler from accredited institution with competition rather than simple recruitment without training. Food handlers should have to update their profession and regular medical screening for communicable diseases.

\section{ACKNOWLEDGEMENTS}

Many thanks to study participants for their time and valuable information. Also thanks for Nebiyu W. for his valuable contribution to the implementation of the study. Thanks to Proceedings of the 8th Africa Nutrition Conference, October 1-5, 2017, Addis Ababa, Ethiopia, which makes the presentation of this paper.

\section{REFERENCES}

[1] World Health organization (WHO), WHO Estimates of The global Burden of Foodborne Diseases Foodborne Disease Burden Epidemiology Reference Group 2007-2015, Editor 2015, WHO: Geneva. p. 10-15.

[2] FAO, The Codex Alimentarius Commission. The General Principles of Food Hygiene. Codex, Rome. 2013.

[3] World Health Organization, Five Keys to Safer Food Manual. World Health Organization,Geneva., F. safety, Editor 2006, WHO: Geneva.

[4] Paez and Ortiz, Food safety practice of food service employee: University of costarica'. 2011: p. 1-6. 
[5] Havelaar, A.H., The First FAO/WHO/AU International Food Safety Conference Addis Ababa, 12-13 February 2019: The public health burden of unsafe foods: a need for global commitment, 2019, WHO, FAO: Addis Ababa. p. 1-3.

[6] Royal College of Physicians. Infected food handlers: occupational aspects of management, a national guideline, London, NHS 2008 plus 2013, ISBN 978-1-86016-335-7.

[7] CarvalhoRossia, p.d.S., et al., Food safety knowledge, optimistic bias and risk perception among food handlers in institutional food services. Food Control, 2017. 73 (Part B): p. 681-688.

[8] Adane M, T.B., Gismu Y, Halefom G, and A. M, Food hygiene and safety measures among food handlers in street food shops and food establishments of Dessie town, Ethiopia: A community-based cross-sectional study. PLoS One, 2018. 13(5): p. e0196919.

[9] Tessema AG, Gelaye KA, and C. DH., Factors affecting food handling practices among food handlers of Dangila town food and drink establishments, North West Ethiopia. BMC Public Health, 2014. 1: p. 571.

[10] Mohammed MA, A.J., Bushra AW, Aljadhey HS. Medications use among pregnan women in Ethiopia: A cross sectional study. Journal of Applied Pharmaceutical Science, 2013; 3 (04): 116-123.

[11] Asrat, M., et al., Assessment of the Sanitary Conditions of Catering Establishments and Food Safety Knowledge and Practices of Food Handlers in Addis Ababa University Students' Cafeteria. Science Journal of Public Health, 2015. 3(2): p. 733-743.

[12] Gizaw Z., Gebrehiwot M., and Teka Z., Food Safety Practice and Associated Factors of Food Handlers Working in Substandard Food Establishments in Gondar Town, Northwest Ethiopia. International Journal of Food Science Nutrition Diet. , 2014. 3(7): p. 138-146.

[13] WHO and FAO, General principles of Food hygiene: Codex alementarius Commision International food standards, 2016, WHO and FAO. p. 5-12.

[14] Jocelyn Andyss, S., The Hawthorne Effect in Hand Hygiene Compliance Monitoring, in Health Service Research2014, University of Toronto.

[15] Oi N.S and S.A. Abdullah, Assessment of Knowledge, Attitudes and Practices (KAP) Among Food Handlers at Residential Colleges and Canteen Regarding Food Safety.Sains Malaysiana. 2011. 40(6): p. 403-410.

[16] Soon JM, Baines R, and Seaman P, Metaanalysis of food safety training on hand hygiene knowledge and Attitudes among Food Handlers. Journal of Food Protection, 2012. 75(4): p. 793-804.

[17] Sihombing, J., R.S. Padmawati, and S.A. Kristina, Knowledge, attitude, and practices regarding food safety among food employees in Ambon City, Indonesia. Mal J Nutr, 2018. 24(2): p. 293-299.

[18] Daru Lestantyo, et al., Safe Food Handling Knowledge, Attitude and Practice of Food Handlers in Hospital Kitchen. International Journal of Public Health Science (IJPHS), 2017. 6(4): p. 324 330.

[19] Thelwell, M.R., Result. Food Safety Knowledge and Self-Reported Practices of Food Handlers in Jamaica, (unpublished doctoral dissertation), University of Walden. (Scholar Works), Minneapolis, Minnesota. U.S.A, 2014.

[20] Rowell AE, et al., The impact of food safety training on employee knowledge of food safety practices for hot/cold self-serve bars Food Protection Trends, 2013. 21 (2): p. 215-221.

[21] Webb M and Morancie A, Food safety knowledge of foodservice workers at a university campus by education level, experience, and food safety training. Food Control, 2015. 50(3): p. 259-264.

[22] Haileselassie M, et al., Food safety knowledge and practices of abattoir and butchery shops and the microbial profile of meat in Mekelle City, Ethiopia. Asian Pac J Trop Biomed., 2013. 3(5): p. 407-12.

[23] Mulugeta K. and Bayeh A., The sanitary condition of food service establishments and food safety knowledge attitude and practice among food handlers in Bahir Dar town. Ethiop J Health Sci., 2012. 22 (1).

[24] Akabanda, F., E.H. Hlortsi, and J. Owusu-Kwarteng, Food safety knowledge, attitudes and practices of institutional food-handlers in Ghana. BMC Public Health, 2017. 17(40).

\section{BIOGRAPHY OF AUTHOR}

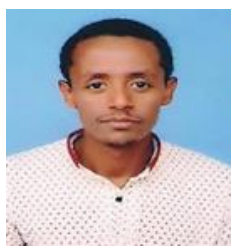

I am Abdu Oumer from Ethiopia. I am currently serving as lecturer and public health researcher at department of public health (nutrition) in higher institution in Ethiopia for the past five years. I have great interest in nutrition researches including malnutrition, clinical nutrition, nutritional intervention, social aspects of nutrition, nutritional epidemiolohgy and others. For more please request me at my email. 